\title{
Investigation of the Prevalence of $C T X-M-1$ Beta-Lactamase Gene in Pseudomonas Aeruginosa Strains Isolated From Urinary Tract Infections in Zanjan Hospitals, Iran
}

\author{
Sasan Dabir, Jamal Mohammadi, Ali Alizadeh, Fatemeh karimi, Khatereh Nori, Sara Mahmoudi, \\ Amir Pournajafi*
}

Department of Biology, Central Tehran Branch, Islamic Azad University, Tehran, Iran

\section{*Corresponding Author}

Amir Pournajafi

\section{Article History}

Received: 13.04 .2020

Accepted: 21.04 .2020

Published: 24.04.2020

\begin{abstract}
Background and Aim: Pseudomonas aeruginosa is an important cause of opportunistic infections. Infection caused by Pseudomonas aeruginosa is often severe and threatening, and is difficult to treat due to its limited susceptibility to antimicrobial agents and resistance to treatment. Therefore, the aim of this study was to identify the molecular identification of $C T X-M-1$ genes in the P. aeruginosa strains isolated from urinary tract infections in Zanjan hospitals. Materials and Methods: In this descriptive-analytical study of the study of 289 cases of urinary tract infection in Zanjan medical centers in 2019, 100 isolates of $P$. aeruginosa were identified by standard bacteriological methods. Antibiotic susceptibility of the isolates was determined by disk diffusion method and ESBL-producing isolates were identified by combined disk method. The bacterial DNA was then extracted and studied by PCR using specific gene primers. Results: The most resistant to ampicillin $(73 \%)$ and tetracycline $(49 \%)$ were the most sensitive to amikacin (90\%) and nitrofurantoin (89\%), respectively. A total of 35 samples were identified as the final ESBL producer. 29 specimens of Pseudomonas bacteria had the CTX-M1 gene. Conclusion: The genes studied in this study were all located on the chromosome of $P$. aeruginosa bacteria. Therefore, further investigation of ESBL genes such as $C T X-M 1$ gene seems necessary to control this bacterium.
\end{abstract}

Keywords: CTX-M1 gene, Extended-Spectrum Beta-Lactamases, Pseudomonas aeruginosa, Urinary Tract Infection, Antibiotic Resistance.

\section{INTRODUCTION}

Since sulfanamides and penicillins have come into the field, a new opportunity has emerged in the treatment of diseases. In the early days of the use of these drugs, numerous epidemics subsided. However, infections caused by infectious organisms remain a serious problem [1]. There are two important mechanisms through which increased resistance to antibiotics and other drugs. The former is due to spontaneous mutation, in the sense that the mutation occurs at a frequency of about 10 to 5\%, altering the susceptibility to the drug, and the drug acts only as a selective agent and promotes the survival of resistant organisms among organisms [2]. The second mechanism of genetic exchange resistance is the genetic information that controls the drug resistance of the bacterium to both chromosomal DNA and extra-chromosomal DNA, ie plasmids, through the transformation, conjugation, and transduction of a (resistant) cell. Transferred to another (sensitive) cell. Hospitalized patients are exposed to nosocomial infections, especially with multidrug-resistant organisms, and are one of the most important contributors to nosocomial infections and as result mortality from Gram-negative bacilli infection. Since antibiotics, especially in ICU wards are usually empirically due to the rush of treatment $[3,4]$. ESBLs, with the power to hydrolyze the wide range of beta-lactam antibiotics used in clinics, pose a serious problem in medicine. Bacteria producing ESBLs with class $\mathrm{C}$ cephalosporinases encoded by the AmpC chromosomal gene have been the most common mechanism of resistance to Gram-negative bacilli against this antibiotic $[5,6]$. Since the second half of the 1980s, with the reporting of variants of ESBLs and the wide geographical distribution of these enzymes, their release has been discussed as an epidemiological phenomenon [7]. The most important ESBLs

Copyright @ 2020: This is an open-access article distributed under the terms of the Creative Commons Attribution license which permits unrestricted use, distribution, and reproduction in any medium for non commercial use (NonCommercial, or CC-BY-NC) provided the original author and source are credited. 
examined are TEM and CTX. CTX was first identified in Germany in 1989 and is divided into five groups, CTX M1, CTX M2, CTXM8, CTXM9 and CTXM15, based on changes in the amino acid sequence. Generally, family members hydrolyze $C T X-M$, cefotaxime, and ceftriaxone better than ceftazidime. They are more inhibited by tazobactam than clavulanic acid $[8,9]$. Urinary tract infections are one of the most common human-acquired infections. In the United States, urinary tract infections are the second most common cause of upper respiratory tract infections, and many men and women are infected throughout their lives. Different factors such as age, sex and immune system influence the prevalence of UTI [10-13]. Pseudomonas aeruginosa is an opportunistic bacterium that often leads to dangerous hospital infestations in people with weakened immune systems. Today, an important problem in the treatment of pseudomonas infections is the high resistance of this bacterium to antibiotics that have no structural or functional similarity to each other [14, 15]. Pseudomonas infections have been reported frequently in burns, urinary tract infections, and lung diseases such as cystic fibrosis. This variation in pseudomonas infections is due to the development of various acquired mechanisms, including gene expression regulation. In addition, by forming biofilm, it provides the ability to protect the host immune system and various antimicrobial agents [16]. P. aeruginosa, gram-negative bacilli, positive oxidase, no spores and aerobic. These bacteria are inherently resistant to penicillin and most beta-lactam antidotes, but are sensitive to the antibiotics ciprofloxacin, tobramycin, and imipenem [17]. The bacterium is clearly seen in burn patients, respiratory patients, and cancer patients undergoing chemotherapy, people with hereditary fibrocystic disease, bacteremia, septicemia, and many other nosocomial infections [18-20]. The aim of this study was to investigate the $C T X-M-1$ gene in the $P$. aeruginosa strains isolated from urinary tract infections in Zanjan.

\section{Materials And Methods}

In this descriptive study, 289 urine samples were collected from outpatients and inpatients of Zanjan hospitals during three months from November to December of 2019 and were cultured on EMB (Merck Company, Germany). Then routine biochemical tests were performed on the colonies. Combined disk test was used to evaluate ESBL producing strains. This experiment was performed using ceftazidime $(30 \mu \mathrm{g})$, cefotaxime $(30 \mu \mathrm{g})$, ceftazidime / clavulanic acid $(30 \mu \mathrm{g} / 10 \mu \mathrm{g})$ and Cefotaxime / clavulanic acid $(30 \mu \mathrm{g} / 10 \mu \mathrm{g})$. For this test, the isolates under study were suspended in physiological saline and their turbidity was adjusted to $0.5 \mathrm{McFarland}$ standards. Then, cotton swabs were cultured in Muller Hinton Agar medium in three directions and after $24 \mathrm{~h}$ incubation at $37^{\circ} \mathrm{C}$, the growth zone diameter was recorded around the discs. Then, cotton swabs were cultured in Muller Hinton Agar medium in three directions and after $24 \mathrm{~h}$ incubation at $37^{\circ} \mathrm{C}$, the growth zone diameter was recorded around the discs. Increase in diameter of more than 5 $\mathrm{mm}$ in diameter growth zone around ceftazidime / clavulanic acid $(30 \mu \mathrm{g} / 10 \mu \mathrm{g})$ and cefotaxime / clavulanic acid (30 $\mu \mathrm{g} /$ $10 \mu \mathrm{g})$ discs compared to ceftazidime $(30 \mu \mathrm{g})$ and cefotaxime $(30 \mu \mathrm{g})$ discs) Indicates ESBL positive of sample and recorded as positive result. In this experiment E. coli ATCC 25922 was used as negative control and E. coli ATCC 35218 as positive control. After confirmation of the presence of $P$. aeruginosa, the antibiogram for the samples was recommended by the Clinical and Laboratory Standards Institute. Antibiotic discs used were tetracycline (30 $\mu \mathrm{g})$, nitrofurantoin $(300 \mu \mathrm{g})$, ceftazidime $(30 \mu \mathrm{g})$, ampicillin sulbactam $(10 \mu \mathrm{g})$, amoxicillin $(25 \mu \mathrm{g})$, amoxicillin-clavulanic $(25 \mu \mathrm{g})$, nalidixic acid $(30 \mu \mathrm{g})$, amikacin $(30 \mu \mathrm{g})$, tobramycin $(10 \mu \mathrm{g})$, imipenem $(10 \mu \mathrm{g})$, ciprofloxacin $(5 \mu \mathrm{g})$ and gentamicin $(10 \mu \mathrm{g})$, (Media Companies). After 24-hour incubation at $37^{\circ} \mathrm{C}$ using a ruler, the growth zone around the discs was measured and compared to the CLSI standards. According to the manufacturer's instructions, the results were based on sensitivity $(\mathrm{S})$ and resistance $(\mathrm{R})$ was reported and semi-susceptible halos were recorded as (I).

After determining the phenotypically positive isolates, the DNA of the identified samples was extracted using kits Oiagen, Hilden (Germany). The PCR reaction was performed with a final volume of $25 \mu 1$, including $1 \mu$ of each primer, Mr. Mix $12.5 \mu \mathrm{l}$, DNA pattern $3.5 \mu \mathrm{l}$ and $7 \mu \mathrm{l}$ of distilled water (all consumables were manufactured by Sinagen Iran). Thermal Cycler device program contains 35 cycles with 4 minute temperature conditions and initial return at $94 \mathrm{C}$, connection at $60 \mathrm{C}$ for 45 seconds, lengthening at $72 \mathrm{C}$ for 1 minute and finally lengthening. The final was done at $72 \mathrm{C}$ for $10 \mathrm{~min}$. The PCR product was then evaluated on $1 \%$ agarose gel with electrophoresis and the gel containing PCR products was placed in a tank containing ethidium bromide for 15 to 20 minutes after the end of the electrophoresis period. The E.coli ATCC 13911 strain with the $C T X-M-1$ gene was used as positive control. Primers used in this study: CTXM1 F:5' ATGGTTAAAAAATCACTGCGTC 3' and R: 5' TTGGTGACGATTTTAGCCGC 3'. In order to statistically analyze the data, the twentieth version of SPSS software and Chi-square test were used. A significant boundary was set at $\mathrm{p}<0.05$.

\section{RESULTS}

In this study, 289 urine samples were collected from 100 (34.60\%) P. aeruginosa. 60 specimens were isolated from the inpatients ward and 40 samples from the outpatients ward. Based on the results of the combined disk test, 40 samples were identified as final ESBL producers. Of the 35 strains of ESBL producing P. aeruginosa, 29 samples had $C T X-M-1$ genes. The results of the sensitivity test against the 12 selected antibiotics are shown in Table 1. 
Table-1: Frequency of antibiotic resistance pattern of $P$. aeruginosa strains isolated from urinary tract infections

\begin{tabular}{|c|r|r|r|}
\hline Antibiotics & Resistance & Intermediate & sensitive \\
\hline Tetracycline & 49 & 10 & 41 \\
\hline Nitrofurantoin & 7 & 4 & 89 \\
\hline Ceftazidime & 29 & 29 & 42 \\
\hline Sulbactam Ampicillin & 73 & 10 & 17 \\
\hline Amoxicillin & 43 & 16 & 41 \\
\hline Amoxicillin-Clavulanic & 45 & 0 & 55 \\
\hline Acid Nalidixic & 30 & 18 & 52 \\
\hline Amikacin & 8 & 0 & 92 \\
\hline Tobramycin & 18 & 2 & 80 \\
\hline Imipenem & 21 & 4 & 75 \\
\hline Ciprofloxacin & 31 & 3 & 66 \\
\hline Gentamicin & 10 & 5 & 85 \\
\hline
\end{tabular}

\section{DisCUSSION}

Broad-spectrum beta-lactamases are a group of beta-lactamase enzymes that are of particular importance in antimicrobial therapy. The rate of ESBL production among Enterobacteriaceae varies worldwide [21]. In the present study, from $100 \mathrm{P}$. aeruginosa isolates, 60 samples from the inpatient ward and 40 samples from the outpatients ward were isolated. Based on the results of the combined disk test, 35 samples were identified as final ESBL producers. 29 specimens of Pseudomonas bacteria had the $C T X-M 1$ gene. The highest resistance to ampicillin $(73 \%)$ and tetracycline $(49 \%)$ were the most sensitive to amikacin (90\%) and nitrofurantoin (89\%), respectively. Wesam et al. have examined the resistance pattern of Pseudomonas to the antibiotics and the results showed that this bacterium has the highest resistance to the antibiotics of nalidixic acid and tetracycline [22]. In another study in Arak in 2013, the resistance of this bacterium to the following antibiotics has been reported: ceftazidime (33.3\%), imipenem (22.2\%), amikacin (20.3\%), ciprofloxacin (15.7\%) and gentamicin (19.4\%) [23]. In the study by Ahadi et al., the resistance of it to imipenem (55\%) and ceftazidime (57\%) has been reported [24]. Rajat Rakesh et al. have reported the resistance of it to the antibiotics of ciprofloxacin $(49 \%)$, gentamicin $(63 \%)$ and imipenem $(14 \%)$ in their study. Chander Anli has reported the resistance of it to the antibiotics of amikacin (25\%), ciprofloxacin (75\%) [25]. Kianpour et al. have reported the resistance of it to the antibiotics of amikacin (58.14\%), ciprofloxacin (42.58\%) and imipenem (14.8\%) [26]. The production of ESBL in the isolates of $P$. aeruginosa is on the rise in the last few years. The rate of tis increase was $20.6 \%$ in Thailand in 2003 [27]. $25.4 \%$ in 2005 in Korea [28], 23.4\% in 2006 in Bolivia and 45.3\% in China in 2006 [29]. In the study by Mirsalehian et al., the production of ESBL in the clinical strains isolated in Tehran, has been reported $40 \%$ which is consistent with the results of present study [30]. Shakibaie et al. [31] have been reported that 41 (34\%) of 120 isolates of $P$. aeruginosa were ESBL-producing strains. Shahcheraghi et al. have been reported that 234 (39\%) of 600 isolates of $P$. aeruginosa were Extended-Spectrum beta-lactamases producing strains [32].

In the study by Shahcheraghi et al. [2010], the presence of the genes of VEB, OXA-10, CTX-M,PER-1,GES1,OXA-1,OXA-4 in the P. aeruginosa strains isolated from the hospitals in Iran has been confirmed (33). Performed in Shiraz, the frequencies of CTX-M1, CTX-M2 and CTX-M3 have been reported 49.9\%, 135\%, 23.1\%, respectively [34]. This difference may be due to the types of samples. In other countries, different results have been reported for the prevalence of ESBL. For example, based on the studies performed in Brazil 2010, the highest prevalence of the enzymes of ESBL was related to CTX-M2 (19.6\%) [35]. In the studies performed in France and Japan, the frequency of the enzyme of CTX-M in the $P$. aeruginosa strains has been reported $0.0 \%$ that is probably due to the appropriate use of beta-lactam antibiotics, especially cephalosporins in these countries [36,37].

\section{Conclusion}

Due to the increased antibiotic resistance among the strains, it is recommended that antibiogram testing be performed before treatment. Also, preventing bacterial strains and therapeutic failures that lead to complication of the infection can be prevented by proper use of existing medicines, completing the course of treatment and avoiding as many antibiotics as possible. Further research in this field will increase our knowledge and more effective exposure to the antibiotic resistance of emerging microorganisms.

\section{REFERENCES}

1. Al Jasser, A. M. (2006). Extended-spectrum beta-lactamase (ESBLS): a global problem.

2. Medeiros, A. A. (1997). Evolution and dissemination of $\beta$-lactamases accelerated by generations of $\beta$-lactam antibiotics. Clinical Infectious Diseases, 24(Supplement_1), S19-S45. 
3. Ensor, V. M., Livermore, D. M., \& Hawkey, P. M. (2007). A novel reverse-line hybridization assay for identifying genotypes of CTX-M-type extended-spectrum $\beta$-lactamases. Journal of Antimicrobial Chemotherapy, 59(3), 387395.

4. Dizaji, A. S., Fathi, R., \& Sales, A. J. (2016). Molecular study of extended-spectrum beta-lactamase (TEM-1) gene in Escherichia Coli isolates collected from Ostad Alinasab Hospital in Tabriz Iran.

5. Jafari-Sales, A., \& Shadi-Dizaji, A. (2018). Molecular analysis of CTX-M genes among ESBL producing in Pseudomonas aeru-ginosa isolated from clinical samples by Multiplex-PCR. HOZAN J Environment Sci, 2(5), 17-29.

6. Sales, A., Fathi, R., \& Mobaiyen, H. (2017). Molecular Study of the Prevalence of CTX-M1, CTX-M2, CTXM3 in Pseudomonas aeruginosa Isolated from Clinical Samples in Tabriz Town, Iran. Electronic J Biol, 13(3), 253-9.

7. Montso, K. P., Dlamini, S. B., Kumar, A., \& Ateba, C. N. (2019). Antimicrobial Resistance Factors of ExtendedSpectrum Beta-Lactamases Producing Escherichia coli and Klebsiella pneumoniae Isolated from Cattle Farms and Raw Beef in North-West Province, South Africa. BioMed Research International, 2019.

8. Jafari Sales, A., Mobaiyen, H., Farshbafi Nezhad Zoghi, J., Nezamdoost Shadbad, N., \& Purabdollah Kaleybar, V. (2014). Antimicrobial resistance pattern of extended-spectrum $\beta$-Lactamases (ESBLs) producing Escherichia coli isolated from clinical samples in Tabriz city, Iran. Adv Environ Biol, 8(16), 179-82.

9. Jafari-Sales, A., Bagherizadeh, Y., Khalifehpour, M., Abdoli-senejan, M., \& Helali-Pargali, R. (2019). Antibiotic resistance pattern and bla-TEM gene expression in Acinetobacter baumannii isolated from clinical specimens of Tabriz hospitals. Zanko Journal of Medical Sciences, 20(65), 20-29.

10. Jafari-Sales, A. (2018). Study of Antibiotic Resistance and Prevalence of bla-TEM gene in Klebsiella pneumoniae Strains isolated from Children with UTI in Tabriz Hospitals. Focus On Medical Sciences Journal, 4(1).

11. Wagenlehner, F. M., Naber, K. G., \& Weidner, W. (2008). Rational antibiotic therapy of urinary tract infections. Medizinische Monatsschrift fur Pharmazeuten, 31(10), 385-90.

12. Jafari Sales, A., \& Mobaiyen, H. (2017). Frequency and resistance patterns in clinical isolates of Escherichia coli Extended Spectrum Beta Lactamase producing treatment Centers in Marand city, Iran. New Cellular and Molecular Biotechnology Journal, 7(26), 19-26.

13. De Francesco, M. A., Ravizzola, G., Peroni, L., Negrini, R., \& Manca, N. (2007). Urinary tract infections in Brescia, Italy: etiology of uropathogens and antimicrobial resistance of common uropathogens. Medical science monitor, 13(6), BR136-BR144.

14. Acton, Q. A. (Ed.). (2012). Pseudomonas Aeruginosa: New Insights for the Healthcare Professional: 2011 Edition: ScholarlyBrief. ScholarlyEditions.

15. Quinn, P. J., Markey, B. K., Leonard, F. C., Hartigan, P., Fanning, S., \& Fitzpatrick, E. (2011). Veterinary microbiology and microbial disease. John Wiley \& Sons.

16. Vanhems, P., Lepape, A., Savey, A., Jambou, P., \& Fabry, J. (2000). Nosocomial pulmonary infection by antimicrobial-resistant bacteria of patients hospitalized in intensive care units: risk factors and survival. Journal of hospital infection, 45(2), 98-106.

17. Rajat, Rakesh, M., Ninama, Govind, L., Mistry, Kalpesh., Parmar, Rosy., Patel Kanu., Vegad, M.M.(2012). National Journal of Medical Research Natl J Med Res, 2(2):156-9.

18. Ryall, B., Davies, J. C., Wilson, R., Shoemark, A., \& Williams, H. D. (2008). Pseudomonas aeruginosa, cyanide accumulation and lung function in CF and non-CF bronchiectasis patients. European Respiratory Journal, 32(3), 740-747.

19. Hacker, J., \& Carniel, E. (2001). Ecological fitness, genomic islands and bacterial pathogenicity. $E M B O$ reports, 2(5), 376-381.

20. Tramper-Stranders, G. A., Van der Ent, C. K., \& Wolfs, T. F. W. (2005). Detection of Pseudomonas aeruginosa in patients with cystic fibrosis. Journal of Cystic Fibrosis, 4, 37-43.

21. Falagas, M. E., \& Karageorgopoulos, D. E. (2009). Extended-spectrum $\beta$-lactamase-producing organisms. Journal of Hospital infection, 73(4), 345-354.

22. Wesam, A. H. (2009). Molecular identification of antibiotics resistant Pseudomonas aeruginosa Wt. Aust J Basic Appl Sci, 3, 2144-53.

23. Taghvaee, R., Shojapour, M., Sadeghi, A., \& Pourbabaie, A. A. (2013). The study of antibiotic resistance pattern and the frequency of extended-spectrum beta-lactamases (ESBL) in pseudomonas aeruginosa strains isolated from medical centers in Arak City, Iran. Qom University of Medical Sciences Journal, 7(4), 36-41.

24. Ahadi, A., Sharif, Zadeh, A., Golshani, Z. (2012). Identification of antibiotic resistance patterns of Pseudomonas aeruginosa isolated from patients admitted with multiple resistances. Journal of Veterinary Laboratory Research. 4(1):119-22.

25. Anil, C., \& Shahid, R. M. (2013). Antimicrobial susceptibility patterns of Pseudomonas aeruginosa clinical isolates at a tertiary care hospital in Kathmandu, Nepal. Asian J Pharm Clin Res, 6(3), 235-8.

26. Kianpour, F., Havaei, S. A., \& Hosseini, M. M. (2010). Evaluation of Pseudomonas Aeroginosa Isolated from Cutaneous Infections and Determination of Drug Resistance Pattern in Patients of Alzahra Hospital in Esfahan. Journal of Isfahan Medical School, 28(110). 
27. Lee, S., Park, Y. J., Kim, M., Lee, H. K., Han, K., Kang, C. S., \& Kang, M. W. (2005). Prevalence of Ambler class A and D $\beta$-lactamases among clinical isolates of Pseudomonas aeruginosa in Korea. Journal of Antimicrobial Chemotherapy, 56(1), 122-127.

28. Celenza, G., Pellegrini, C., Caccamo, M., Segatore, B., Amicosante, G., \& Perilli, M. (2006). Spread of bla CTX-Mtype and bla PER-2 $\beta$-lactamase genes in clinical isolates from Bolivian hospitals. Journal of Antimicrobial Chemotherapy, 57(5), 975-978.

29. Strateva, T., Ouzounova-Raykova, V., Markova, B., Todorova, A., Marteva-Proevska, Y., \& Mitov, I. (2007). Problematic clinical isolates of Pseudomonas aeruginosa from the university hospitals in Sofia, Bulgaria: current status of antimicrobial resistance and prevailing resistance mechanisms. Journal of medical microbiology, 56(7), 956-963.

30. Owlia, P., Saderi, H., Karimi, Z., Rad, A., Bagher, S. M., \& Bahar, M. A. (2008). Phenotypic detection of Metallobeta-Lactamase producing Pseudomonas aeruginosa strains isolated from burned patients. Iranian Journal of Pathology, 3(1), 20-25.

31. Shakibaei, M., \& Shah, C. F. (2013). Detection of TEM, SHV and PER type extended-spectrum $\beta$-lactamase gene among 19-Goudarzi H, Aghamohammad S, Hashemi A, Nikmanesh B, Noori M. Distribution of blaTEM, blaSHV and bla CTX-M genes among Escherichia coli isolates causing urinary tract infection in children. Arch Clin Infect Dis, 8, e1b207.

32. Shahcheraghi, F., Nasiri, S., \& Noveiri, H. (2010). The Survey of Genes Encoding Beta-Lactamases, in Escherichia Coli Resistant to Beta-Lactam and Non-Beta-Lactam Antibiotics. Iranian Journal of Basic Medical Sciences, 13(1), 230-237.

33. Shahcheraghi, F., Shakibaie, M., \& Noveiri, H. (2010). Molecular identification of esbl genes blages-1, blaveb-1, blactx-m blaoxa-1, blaoxa-4, blaoxa-10 and blaper-1 in pseudomonas aeruginosa strains isolated from burn patients by pcr, rflp and sequencing techniques.

34. Kownani, M., Baserisalehi, M., \& Bahador, N. (2013). Frequency of Extended Spectrum $\beta$-Lactamase Genes in Pseudomonas Aerugionsa Isolates from Shiraz Hospital, Iran. Journal of Isfahan Medical School, 31(243).

35. Jafari-Sales, A., \& Shadi-Dizaji, A. (2018). Molecular analysis of CTX-M genes among ESBL producing in Pseudomonas aeru-ginosa isolated from clinical samples by Multiplex-PCR. HOZAN J Environment Sci, 2(5), 17-29.

36. Naas, T., Cuzon, G., Truong, H., Bernabeu, S., \& Nordmann, P. (2010). Evaluation of a DNA microarray, the Check-Points ESBL/KPC array, for rapid detection of TEM, SHV, and CTX-M extended-spectrum $\beta$-lactamases and KPC carbapenemases. Antimicrobial agents and chemotherapy, 54(8), 3086-3092.

37. Yamasaki, K., Komatsu, M., Yamashita, T., Shimakawa, K., Ura, T., Nishio, H., ... \& Aihara, M. (2003). Production of CTX-M-3 extended-spectrum $\beta$-lactamase and IMP-1 metallo $\beta$-lactamase by five Gram-negative bacilli: survey of clinical isolates from seven laboratories collected in 1998 and 2000, in the Kinki region of Japan. Journal of Antimicrobial Chemotherapy, 51(3), 631-638. 